\title{
Estimating User Credibility in Multimedia Information Flows
}

\author{
Alexandru Lucian Ginsca \\ CEA, LIST \\ Vision \& Content Engineering Laboratory \\ Gif-sur-Yvette, France \\ alexandru.ginsca@cea.fr
}

\begin{abstract}
We outline in this paper the problem of determining user credibility in a multimedia setting. We briefly describe the context of our research and present initial findings for user credibility estimates and their applications both in a Community Question Answering and Image Retrieval scenario. We also present the main directions for future work.
\end{abstract}

Credibility, User modeling, Re-ranking, Image Retrieval, Community question answering

\section{INTRODUCTION}

When searching for information, offline or online, one of the main questions is how credible the information is. Credibility is an important aspect in information retrieval and extraction tasks. While it was thoroughly studied for offline sources of information, for Web 1.0 sites Metzger (2007) and for blogs (and integrated in successful information retrieval algorithms) Weerkamp and de Rijke (2012), there is little work dealing with credibility of multimedia flows shared on social networks.

We plan to explore credibility in the context of social networks, where Web sites are replaced by users as central concepts of the network structure. In this context, one of our main research questions is how to determine how credible a user is based on his online profile and contributions in the community? This question can be further expanded into several more focused interest points. We are interested to investigate which dimensions of a user's online representations are most relevant for mining his credibility, if it is possible to combine multiple dimensions in order to improve the mining process, if credibility mining can be deployed at large scale and how can user credibility scores be used in other applications, such as a re-ranking method in an information retrieval system. A first valuable resource for our studies is represented by the question answering communities where introducing a credibility component could provide a fast indicator for selecting the better answers. Since information streams provided by Web 2.0 users are formed of different types of media, another choice to test our approach to credibility mining is on Flickr data where credibility is derived using a combination of structural factors and content analysis.

\section{FIRST RESULTS}

In this section, we briefly describe our initial findings both on the Flickr and StackOverflow communities.

\subsection{Community Question Answering}

Our first work direction is oriented towards Community Question Answering in which we cover credibility from an expertise perspective. Our objective here is to determine if there are any particular dimensions of a user's profile or activity in the community that can be exploited to spot high quality answers. We first perform an in-depth analysis of the information provided by the users in their profiles in order to discriminate features that are correlated to expertise. Then, we propose an answer ranking scenario in which we assess the predictive capabilities of profile and activity features. In our experiments, we use a large scale corpus from StackOverflow, a very active Q\&A community focused on technical topics. The main contributions of our research can be summarized as follows:

- We improved the understanding of CQA content through a detailed user profile analysis. We found interesting correlations between answer quality and features derived from the user's profile (type of username, personal 
website, age, location, number of votes given etc.). As expected, the more complete a profile is, the higher the chances are for that user to provide good quality answers. However, not all features are equally discriminant and we demonstrate that user name, user location and personalized avatars are the most useful ones in an answer ranking task. We also found that a classifier using a language model of users' descriptions can be used to highlight good answers.

- We proposed novel automatic answer ranking methods. Different configurations based on user profile information and/or user activity were tested. We showed that an appropriate combination of the two types of data gives the most accurate results. A $22 \%$ performance boost is obtained compared to a ranking based on the temporal order of the answers.

\subsection{Image Retrieval}

We started the work in this direction by analyzing possible sources for credibility estimations in the Flickr community. In a first series of experiments, we analyzed the correlations between user related features and the relevance of the tags associated with the photos. The relevance of the tags is a binary value taken from the ground truth built for the ImageCLEF 2011 Wikipedia retrieval track. Tsikrika et al. (2011) We tested over 20 features related to the user's profile(e.g. username, website), his social network(e.g. the number of groups he is part of, the number of contacts) or his activity in Flickr(e.g. the average number of tags per photo or the average upload time between any two consecutive uploads). We found that all this features had a weak correlation with the binary relevance score. Out of them the most informative proved to be the number of tags of the photo. We also performed information gain feature selection on this set of features and the results were similar with those from the correlation tests.

In order to test if a more complex model would capture a relationship between user features and the global relevance of the user's photos in the retrieval process, we have translated the problem into a supervised regression one. For each user, we assigned a score that represents the number of photos that were posted by that user marked as "correct" in the evaluation corpus over the total number of photos posted by the user that are found in the evaluation corpus. We tested several classifiers and performed a 10 fold cross-validation evaluation. We obtained an error rate over the 10 splits of 0.427 for a SVM classifier and of 0.413 for a Random Forests classifier for the best selection of parameters. We also tested the impact of the predicted scores in the retrieval task. For each split file from the regression scenario containing a set of users, we generated a baseline ranking of the images based on the initial ordering in the gold file. We then performed a re-ranking based on the predicted scores of each classifiers. We have observed an average increase of the MAP score over the 10 splits of $3.25 \%$ when using the scores predicted by the SVM model and of $3.8 \%$ when using Random Forests. One possible explanation for these results is the nature of the test corpus that was used, in which the vast majority of the users have only one or two images in the initial gold corpus and this is a weak indicator of the global user's behavior that we are trying to capture. To resolve this problem, we built a new balanced evaluation corpus in which a user is represented by at least 20 photos but the analysis on this corpus is left for future work.

\section{FUTURE WORK}

The main direction for future work is continuing the study on the use of credibility estimations in the Flickr community and their impact on a retrieval task. An important aspect of the Flickr community that has not yet been explored is the social component. This can be reflected in the comments that a user receives for his photos or a more detailed analysis of the groups that that the user is part of. Group activity can uncover the user's involvement in the community as well as it can be an indicator of the topics that are most related to the user's activity. Another aspect that interests us is the use of visual information in the credibility models. A first approach is to derive a specificity score from the users tagging behavior in report to the images (represented by visual descriptors). This can be interpreted as how a certain user associates tags with images differs from the global associations of a large group of users from the Flickr community.

\section{REFERENCES}

Metzger, M. J. (2007). Making sense of credibility on the web: Models for evaluating online information and recommendations for future research. Journal of the American Society for Information Science and Technology, 58(13):2078-2091.

Tsikrika, T., Popescu, A., and Kludas, J. (2011). Overview of the wikipedia image retrieval task at imageclef 2011. In CLEF (Notebook Papers/LABs/Workshops): CLEF.

Weerkamp, W. and de Rijke, M. (2012). Credibilityinspired ranking for blog post retrieval. Information retrieval, pages 1-35. 